\title{
Screening the elderly
}

\author{
Charles B Freer
}

One of the many controversial features of the government's revised general practitioner contract is the preventive protocol required to satisfy the enhanced annual capitation fee for patients aged 75 and over. One possible justification for the government's desire to encourage more anticipatory care for older people has been the fact that despite 40 years of interest and research activity in geriatric screening in Britain only a minority of practices have included such screening in their range of services. There are several explanations for this.

As with other care professionals, general practitioners may not see older people as the most interesting or challenging group of patients. Also, general practitioners have legitimate concerns about the implications of universal screening of older people for their workload, ${ }^{2}$ and many have yet to be convinced about the feasibility and effectiveness of screening. ${ }^{3}$ It seems likely that screening can reduce mortality and the use of resources, but it remains doubtful if it is realistic to expect to find the motivation, time, and resources for research studies in every practice in the country.

Of fundamental concern is the government's overly reductionist view of the process of care in general practice, which underestimates and undervalues the preventive content of good routine care. But whatever the academic arguments about screening, since 1 April 1990 general practitioners have been required to offer patients aged 75 and over the services shown in the box.

\section{Implementing the new contract}

Faced with the wide ranging administrative and workload implications of the new contract, the general practitioner may find the prospect of having to carry out home based screening of all patients aged 75 years and over every year daunting, especially for those practices with above average numbers of older people. At the same time it is important not to overestimate the challenge.

The requirement to provide an annual health check need not be a complex or time consuming task. Too often the screening protocols that have been published are fairly detailed assessments. Furthermore, most older people are reasonably fit and well, and the important but relatively small number of frail and dependent older people is likely to be well known to the practice team. It is possible to give constructive and positive guidance on how the contract proposals relating to older patients can be introduced.

\section{Overall strategy}

Some practices, in particular those with a special interest in the care of older people, may opt for a separate screening programme for all their elderly patients over and above their routine care. Consideration of existing patterns of care suggests, however, that most practices may not find it necessary to commit the time and resources required for comprehensive screening programmes. Ninety per cent of patients in this age group see their family doctor at least once a

\section{Services for patients aged 75 and over}

A home visit at least annually to see the home environment and to find out whether carers and relations are available

Social assessment (lifestyle, relationships)

Mobility assessment (walking, sitting, use of aids)

Mental assessment

Assessment of the senses (hearing and vision)

Assessment of continence

General functional assessment

Review of medication

year; the average annual consultation rate is six, and more than half the contacts are in the patient's home. ${ }^{45}$ There are clear attractions in completing screening during some of these frequent contacts with older patients - so called opportunistic or consultation based screening. ${ }^{6}$ Even in surgery consultations most of the required information can be collected, and then the person can be offered a home visit as required by the contract. Many are likely to decline this. With this approach to screening, those patients on the practice list not seen during routine contacts in any yearprobably as few as $10 \%$ of those over 75 -could be visited at home or offered a screening visit by letter. Again it is likely that some will decline this invitation.

Both universal and consultation based approaches will require an up to date age-sex register and some method, manual or computerised, to record that screening has been completed (or declined) for that year.

\section{The screening schedule}

The high frequency of contact between general practitioners and their older patients probably means that much of the information required by the new contract will be known; what is in fact required is a format to record it in the patient's notes. Special interest journals and a number of drug companies are already producing relevant recording systems. No one system will suit all practices, but as the main objective of screening is to identify those people who require assessment, a more detailed procedure, the schedule chosen should be simple and brief. Using a simple, brief schedule is essential if consultation based screening is to be feasible. There is also broad agreement that screening should be oriented to the patient's functioning and not to disease; hence testing of blood and urine is not indicated.

British general practitioners need no instruction in home and social assessment, but the table shows how mobility, the senses, continence, and general function can be assessed. For example, a functional screening approach to mobility state would first of all simply mean establishing if there was any actual problem with mobility. Most older people will have satisfactory functional mobility. For those with problems the types of functions shown in the table will help to establish the nature of any limitations. It is then possible to
Charles B Freer, MRCGP, general practitioner

BrMed f 1990;300:1447-8 
determine the cause of limited mobility: osteoarthritis of hips or knees, chiropody problems, etc. In contrast, disease oriented or problem oriented screening would require a long checklist of problems to be gone through $-\mathrm{a}$ much more time consuming method. It is also inefficient as it will almost certainly create a long list of problems - that is, apparent unmet needs when in reality many of these problems are of minimal functional importance. In terms of chiropody it is less important to determine the number of older people with corns and bunions than those who are experiencing difficulties with footwear or walking because of chiropody problems.

Criteria for functional assessment of patients aged over 75

\begin{tabular}{llll}
\hline Mobility & \multicolumn{1}{c}{ Senses } & \multicolumn{1}{c}{ Continence } & General function \\
\hline Gait & Problems with conversation or television & Frequency & Cooking \\
Negotiating stairs & Ear wax & Dysuria & Diet \\
Rising from chair & Use of hearing aid & Stress incontinence & Shopping \\
Transferring & Able to read newspapers & Prostatic symptoms & Dressing \\
Balance & Recognises faces across the room & Pattern of bowel function & Bathing \\
Use of aids & State of glasses & Recent changes & Toileting \\
& & Laxative use & \\
& & Faecal staining & \\
& & &
\end{tabular}

The lack of available treatment for some of the problems likely to be detected-for example, the socioeconomic deprivation of many older people-fails to meet one of the criteria to be satisfied when introducing screening.? It may not be too idealistic, however, to believe that the systematic collection of this type of information could produce a powerful statement on the unmet medical and social needs of older people in Britain and lead to a more appropriate distribution of available resources. To a large extent this will depend on the impact of other current legislative changes relating to community care.

A review of medication is a particularly relevant feature of good practice in the care of older people and should consider the need for continued treatment, the presence of side effects and interactions, the costs of treatment, and the use of non-prescription drugs. It also provides the opportunity to assess compliance and the need to provide help in understanding and following drug regimens.

The prevalence of depression and dementia among older people justifies the inclusion of assessment of mental state in screening. Depression is common, frequently missed, and can often be treated effectively. For the detection of dementia several short mental state questionnaires are in common use, and the type of questions included are shown in the box. Three or more incorrect answers suggest intellectual deterioration, and the number of errors indicates the severity. Williamson's functional case finding protocol provides a more detailed framework for the sociomedical screening of older people. ${ }^{8}$

It needs to be emphasised that brief functional and mental assessments cannot have complete validity, and therefore false positive and false negative results are to be expected. Indeed, even more detailed assessments do not have $100 \%$ specificity and sensitivity.

\section{Who should screen?}

The new contract does not require screening to be done by the doctor, but as already discussed there is an important potential for opportunistic screening. Health visitors and practice nurses can also carry out screening during routine contacts. In larger practices and particularly those with above average numbers of older people it may be necessary to involve existing members of the team or perhaps to engage additional practice nurse time to ensure that all eligible and willing patients are screened. The capitation fee for the 75 and over age group has been increased by $120 \%$, and

\section{Short questionnaire to determine mental state}

1 Today's date

2 Day of week

3 Age

4 Address

5 Year

6 Date of birth

7 Year of first world war

8 Name of monarch or prime minister

9 Count backwards from 20 to one

this should go some way to subsidising any required increase in staff resources.

\section{Conclusion}

The manner in which these and other contract changes were introduced has led to an unfortunate loss of good will on the part of many family doctors. In particular, the changes seem to underestimate the commitment of many British general practitioners to good care of older people, including preventive care. Moreover, most older people are reasonably healthy, well, and content, so that imposing universal domiciliary screening on an annual basis seems to be a wasteful use of limited resources and to be inconsistent with the principle of targeting support to those with greatest needs, which is fundamental to the government's other legislation on community care. Whatever criticisms can be directed at the government for its insistence on annual domiciliary screening of all people aged over 75 , it has to be said that the areas covered by the contract provide a reasonable framework for a sociomedical profile of older patients and the elderly population.

It is important that general practitioners support an anticipatory dimension to the care of older people, but at the same time those who administer general practice need to recognise that variation in general practice is not only a reality but can also be a strength. The emerging local dialogue between family practitioner committees, health boards, and general practitioners may mean that the contract changes will be introduced with more pragmatism than rigidity and that monitoring of the contract's operation and impact can be used to make the changes that experience and evidence dictate.

1 Barber $\mathrm{JH}$, Wallis JB. The effects of a system of geriatric screening an assessment on general practice workloads. Health Bulletin 1982;40:125-32. Barley S. An uncompromising report on health visiting for the elderly. Br Med $\mathcal{f}$ 1987;294:595-6.

3 Freer $\mathrm{CB}$. Geriatric screening: a reappraisal of preventive strategies in the care of the elderly. $\mathcal{F}$ R Coll Gen Pract 1985;35:288-90.

4 Office of Population Censuses and Surveys. Britain's elderly population. Census guide 1. London: HMSO, 1984

5 Royal College of General Practitioners. Morbidity statistics from general practice 1981-82. Third national survey. London: HMSO, 1986.

6 Freer $\mathrm{CB}$. Consultation-based screening of the elderly in general practice: a pilot study. I R Coll Gen Pract 1987;37:455-6.

7 Wilson JMG, Jungner G. Principles and practice of screening for disease. Geneva: World Health Organisation, 1968 .

8 Williamson J. Prevention, screening and case finding - an overview. In: Taylor RC, Buckley EG, eds. Preventive care of the elderly: a review of curren developments. London: Royal College of General Practitioners, 1987:45. (Occasional paper No 35 .

\section{Correction}

Decontamination of instruments and control of cross infection in general practice

A printers' error occurred in this practice observed article by $\mathrm{Mr}$ D R Morgan and others (26 May, p 1379). In the second paragraph of the discussion a previous report had indicated that only $8 \%$ of practices had autoclaves and not $80 \%$ as published. 